\title{
Can we predict neurological outcome following out- of-hospital cardiac arrest (OHCA) and therapeutic hypothermia?
}

\author{
Khusrav Bajan
}

Out-of-hospital cardiac arrest is associated with a poor outcome. Despite improvement in postresuscitation Intensive Care Unit Care, severe neurologic damage due to hypoxic brain insult still remains a major concern, in more than $50 \%$ of the OHCA survivors. ${ }^{[1]}$

Early neuro-prognostication in postcardiac arrest comatose survivors is crucial to help give appropriate information to the relatives, especially about decisions to limit or withdraw life-support measures.

Therapeutic hypothermia has emerged as the standard of care in comatose post-cardiac arrest survivors in the last decade as a modality to improve neurological outcome $^{[2]}$ although disputed in the recent literature. This has led to its incorporation in the 2010 American Heart Association postcardiac arrest care guidelines.

In the era of therapeutic hypothermia, early neuroprognostication is challenging. The sedation and neuromuscular blockade used during hypothermia significantly influences clinical examination. There can be significant increase in serum concentrations of sedatives and neuromuscular blockers, owing to hypothermia-induced alteration in pharmacodynamics and pharmacokinetics, thus questioning the reliability of clinical signs. ${ }^{[3]}$

Brain stem reflexes, especially the corneal and pupillary light reflexes are assessed daily in post-cardiac arrest comatose survivors. However, again, these reflexes are

\section{From:}

Dr. Khusrav Bajan

Consultant Physician \& Intensivist, Department of Medicine and Critical

Care, PD Hinduja Hospital \& Medical Research Centre, Veer Savarkar Marg,

Mahim, Mumbai-400 016, India.

\section{Correspondence:}

Consultant Physician \& Intensivist, Department of Medicine and Critical Care, PD Hinduja Hospital \& Medical Research Centre, Veer Savarkar Marg,

Mahim, Mumbai-400 016, India.. E-mail: drkhusrav@gmail.com

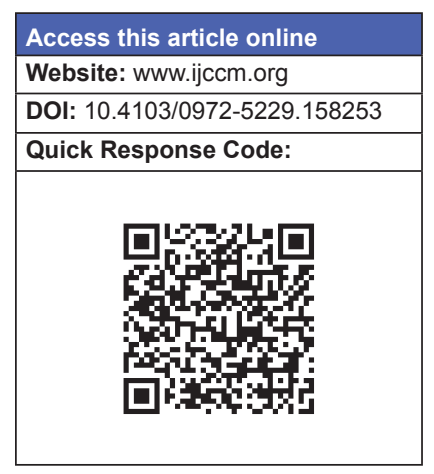

difficult to assess reliably, as these can be altered by sedation used during hypothermia. During rewarming, when sedation is weaned, if there is the good motor response (Glasgow Coma Scale-motor [GCS-M] $\geq$ or 5), it indicates favorable neurological outcome and no further predictors are needed. On the other hand, if the patient continues to be unresponsive with the poor motor response (GCS $\leq 4)$, then prognosis worsens each day, and other prognostic tools are needed. When GCS-M $\leq 2$ was considered alone at day 3, the false positive rate for poor outcome was found to be $12-24 \%{ }^{[7]}$ There is no reliable data to define optimal timing to perform a neurological examination to neuro-prognosticate in post-cardiopulmonary resuscitation patients treated with hypothermia.

The Glasgow-Pittsburgh cerebral performance categories (CPC) scale is commonly used as an outcome measure for neurological recovery. In the study conducted by Nicholas et al, patients admitted following OHCA treated with

This is an open access article distributed under the terms of the Creative Commons Attribution-NonCommercial-ShareAlike 3.0 License, which allows others to remix, tweak, and build upon the work non-commercially, as long as the author is credited and the new creations are licensed under the identical terms.

For reprints contact: reprints@medknow.com

How to cite this article: Bajan K. Can we predict neurological outcome following out-of-hospital cardiac arrest (OHCA) and therapeutic hypothermia? Indian J Crit Care Med 2015;19:301-3. 
hypothermia were evaluated for neurological outcome using the СРC scale and a score between 3 and 5 was considered to have an unfavorable neurological outcome at hospital discharge. ${ }^{[4]}$

In this study, status myoclonus at $<24 \mathrm{~h}$, absent brain stem reflexes and motor response worse than flexion at day 3, were noted to be reliable clinical predictors of unfavorable outcome.

Myoclonus is a common event in postcardiac arrest victim. In a study, posthypoxic myoclonus was reported in $20 \%$ of patients, out of which $9 \%$ had a good outcome. ${ }^{[5]}$ Before the advent of hypothermia, myoclonus was considered as an early clinical predictor of poor outcome. However, with hypothermia, a manifestation of myoclonus is usually suppressed by sedatives and muscle relaxants and hence myoclonus cannot be considered as a sign of poor outcome. ${ }^{[6]}$ There are case reports of isolated patients who developed myoclonus during hypothermia but regained consciousness. ${ }^{[9]}$

In a survey conducted by Friberg et al. amongst the members of European Society of Intensive Care Medicine (ESICM), it was confirmed that no index predicts poor outcome with absolute certainty and hence a multimodality approach appears to be the best way to reduce the risk of a falsely pessimistic prediction. ${ }^{[8]}$

As per the current evidence, only clinical examination to determine poor neurological outcome is not adequate and supplementation by other modalities such as electro encephalography (EEG), somatosensory evoked potentials (SSEP), biomarkers, neuroimaging are essential to increase accuracy for neuro-prognostication.

Electro encephalography detects nonconvulsive status epilepticus and specific EEG patterns are used to predict outcomes. Early nonconvulsive seizures are associated with poor outcome. On the other hand, a good outcome has been reported in seizures occurring during rewarming phase treated aggressively with antiepileptics. ${ }^{[10]}$ Dynamic changes in EEG called "reactive EEG" are strongly associated with arousal as they reflect intact reticular activating system and a negative EEG predicts a poor outcome. ${ }^{[7]}$ EEGs are considered to be of the malignant pattern if post hypoxic status epilepticus, alpha coma, burst suppression or generalized suppression is evident and associated with poor prognosis.

In a study by Fugate et al., a malignant EEG pattern was associated with a $0 \%$ false positive rate to predict neurological outcome. ${ }^{[11]}$
In patients with hypoxic ischemic encephalopathy after cardiac arrest SSEPs can be used for prognostication. Bilateral absent SSEPs at rewarming phase are associated with poor outcome with $0 \%$ false positive rate. ${ }^{[7]}$

Biomarkers of brain injury in comatose survivors from cardiac arrest are serum Neuro Specific Enolase (NSE) and S-100 $\beta$ levels. Though hypothermia reduces serum NSE levels, a single high NSE value on day 3 after hypothermia predicts poor outcome with false positive rate ranging from $7 \%$ to $29 \%$, and to reduce false positives, we need much higher cut-off values $(>50-80 \mu \mathrm{g} / \mathrm{L}) \cdot{ }^{[12]}$

As per the current guidelines, limited data are available to support the use of computed tomography (CT) for neuro-prognostication in comatose post cardiac arrest patients. However, early CT brain is useful to rule out a cerebral cause of coma/cardiac arrest, more so in patients with preceding neurological symptoms. ${ }^{[13]}$

In conclusion, neuro-prognostication is a challenging task, particularly in this era of therapeutic hypothermia. To predict the neurological outcome accurately in comatose cardiac arrest survivors treated with hypothermia, a multimodal approach is recommended. Though there is no optimal timing, the prognostication should be done only 72-96 $\mathrm{h}$ after cardiac arrest. The clinical neurological examination is the gold standard, but other modalities are required for increasing accuracy, reducing false positive rates and avoiding early pessimistic prediction. We need further studies to increase the accuracy for neuroprognostication in patients with hypoxic-ischemic insult but for now, as per the evidence, European Resuscitation Council and ESICM have issued guidelines, which included four main prognostic tools: (1) Clinical examination, (2) EEG, (3) biomarkers (4) imaging studies. ${ }^{[13]}$

In my opinion, a multimodal approach is the way to go, especially to avoid premature and inappropriate negative predictions in patients treated with hypothermia.

\section{Financial support and sponsorship}

Nil

\section{Conflicts of interest}

There are no conflicts of interest.

\section{References}

1. Target temperature management at 33 versus 36 degrees after cardiac arrest. N Engl J Med 2013; 369:2197-2206.

2. Hypothermia after Cardiac Arrest Study Group. Mild therapeutic hypothermia to improve the neurologic outcome after cardiac arrest. N Engl J Med 2002;346:549-56. 
3. Arpino PA, Greer DM. Practical pharmacologic aspects of therapeutic hypothermia after cardiac arrest. Pharmacotherapy 2008;28:102-11.

4. Blondin NA, Greer DM. Neurologic prognosis in cardiac arrest patients treated with therapeutic hypothermia. Neurologist 2011;17:241-8.

5. Bouwes A, van Poppelen D, Koelman JH, Kuiper MA, Zandstra DF, Weinstein HC, et al. Acute posthypoxic myoclonus after cardiopulmonary resuscitation. BMC Neurol 2012;12:63.

6. Taccone F, Cronberg T, Friberg H, Greer D, Horn J, Oddo M, et al. How to assess prognosis after cardiac arrest and therapeutic hypothermia. Crit Care 2014;18:202.

7. Rossetti AO, Oddo M, Logroscino G, Kaplan PW. Prognostication after cardiac arrest and hypothermia: a prospective study. Ann Neurol 2010;67:301-7.

8. Friberg H, Cronberg T, Dünser MW, Duranteau J, Horn J, Oddo M. Survey on current practices for neurological prognostication after cardiac arrest. Resuscitation 2015;90:158-62

9. Hovland A, Nielsen EW, Klüver J, Salvesen R. EEG should be performed during induced hypothermia. Resuscitation 2006;68:143-6.

10. Rossetti AO, Oddo M, Liaudet L, Kaplan PW. Predictors of awakening from postanoxic status epilepticus after therapeutic hypothermia. Neurology 2009;72:744-9.

11. Fugate JE, Wijdicks EF, Mandrekar J, Claassen DO, Manno EM, White RD, et al. Predictors of neurologic outcome in hypothermia after cardiac arrest. Ann Neurol 2010;68:907-14.

12. Daubin C, Quentin C, Allouche S, Etard O, Gaillard C, Seguin A, et $a l$. Serum neuron-specific enolase as predictor of outcome in comatose cardiac-arrest survivors: A prospective cohort study. BMC Cardiovasc Disord 2011;11:48.

13. Sandroni C, Cariou A, Cavallaro F, Cronberg T, Friberg H, Hoedemaekers C, et al. Prognostication in comatose survivors of cardiac arrest: An advisory statement from the European Resuscitation Council and the European Society of Intensive Care Medicine. Resuscitation 2014;85:1779-89. 